\title{
Discordância entre Diretrizes Internacionais sobre Critérios de Prevenção Primária de Morte Súbita na Cardiomiopatia Hipertrófica
}

\author{
Discrepancy between International Guidelines on the Criteria for Primary Prevention of Sudden Cardiac Death \\ in Hypertrophic Cardiomyopathy
}

\author{
Beatriz Piva e Mattos, ${ }^{1,2}$ Fernando Luís Scolari, ${ }^{1,2}$ Henrique lahnke Garbin ${ }^{2} \bullet$ \\ Hospital de Clínicas de Porto Alegre, ${ }^{1}$ Porto Alegre, $R S$ - Brasil \\ Universidade Federal do Rio Grande do Sul - Faculdade de Medicina, ${ }^{2}$ Porto Alegre, RS - Brasil
}

\section{Resumo}

Fundamento: A estratificação de risco para morte súbita (MS) na cardiomiopatia hipertrófica (CMH) baseia-se em algoritmos distintos propostos pela diretriz norte-americana, ACCF/AHA 2011 e europeia, ESC 2014.

Objetivo: Analisar o modelo ESC 2014 na determinação do risco de MS e indicação de cardiodesfibrilador implantável (CDI) em prevenção primária na CMH por meio de confrontação com a normativa norte-americana.

Métodos: Foi avaliada uma coorte de pacientes com CMH, calculado o escore ESC HCM-Risk-SCD e analisada a concordância dos critérios de indicação de CDI entre as duas diretrizes pelo coeficiente de Kappa. O nível de significância adotado nas análises estatísticas foi de $5 \%$.

Resultados: Em 90 pacientes consecutivos, seguidos por $6 \pm 3$ anos, o escore calculado foi de $3,2 \pm 2,5 \%$. Os preditores que mais contribuíram para o cálculo nas faixas de baixo $(1,88 \%[1,42-2,67])$, médio $(5,17 \%[4,89-5,70])$ e alto risco $(7,82 \%[7,06-9,19])$ foram espessura parietal máxima do ventrículo esquerdo $(1,60 \%[1,25-2,02] ; 3,20 \%[3,18-3,36]$; $4,46 \%[4,07-5,09])$, diâmetro do átrio esquerdo $(0,97 \%[0,83-1,21] ; 1,86 \%[1,67-2,40] ; 2,48 \%[2,21-3,51])$ e idade $(-0,91 \%[0,8-1,13] ;-1,90 \%[1,12-2,03] ;-2,34 \%[1,49-2,73])$. O modelo europeu reduziu as recomendaçóes de CDI em $32(36 \%)$ pacientes. Entre os $43(48 \%)$ em classe Ila pela $A C C F / A H A, 8(18 \%)$ migraram para IIb e $24(56 \%)$ para III. Baixa concordância foi identificada entre as duas sistematizações, Kappa $=\mathbf{0 , 3 5 5 , p}=\mathbf{0 , 0 0 0 1}$. Dos $8(9 \%)$ pacientes com MS ou choque apropriado, $4(50 \%)$ atingiram indicação lla pela $A C C F / A H A$, mas nenhum pela $E S C$.

Conclusão: Baixa concordância foi identificada entre as diretrizes analisadas. $O$ novo modelo reduziu as indicações de CDI, notadamente em classe Ila, mas deixou desprotegida a totalidade de pacientes com MS ou choque apropriado. (Arq Bras Cardiol. 2020; 115(2):197-204)

Palavras-chave: Cardiomiopatia Hipertrófica/genética; Hereditariedade; Morte Súbita Cardíaca; Arritmias Cardíacas; Síncope; Desfibriladores Implantáveis; Choque; Estudos de Coortes.

\begin{abstract}
Background: Risk stratification for sudden cardiac death (SCD) in hypertrophic cardiomyopathy (HCM) is based on different algorithms proposed by the 2011 ACCF/AHA and 2014 ESC guidelines.

Objective: To analyze the 2014 ESC model for SCD risk stratification and primary prevention ICD (implantable cardioverter defibrillator) in HCM in comparison to the North American guideline.

Methods: An HCM cohort was evaluated and the ESC HCM-Risk SCD score was calculated. Agreement of ICD recommendations Criteria between the two guidelines was analyzed with Kappa coefficient. $P<0.05$ was adopted for the statistical analysis.

Results: In 90 consecutive patients followed for $6 \pm 3$ years, the mean calculated ESC risk score was $3.2 \pm 2.5 \%$. The risk predictors that have mainly contributed to the score calculation in the low (1.88\% [1.42-2.67]), intermediate (5.17\% [4.89-5.70]) and high-risk (7.82\% [7.06-9.19]) categories were: maximal left ventricular wall thickness (1.60\% [1.25-2.02]; 3.20\% [3.18-3.36]; 4.46\% [4.07-5.09]), left atrial diameter (0.97\% [0.83-1.21]; 1.86\% [1.67-2.40]; 2.48\% [2.21-3.51]) and age (-0.91\% [0.8-1.13]; -1.90\% [1.12-2.03]; -2.34\% [1.49-2.73]). The European model decreased the ICD recommendations in 32 (36\%) patients. Among the 43 (48\%) individuals with class Ila recommendation under the 2011 ACCF/AHA guideline, 8 (18\%) were downgraded to class IIb and 24 (56\%) to class III. Low agreement was found between the two systems: Kappa $=0.355$ and $p=0.0001$. In 8 (9\%) patients with SCD or appropriate shock, 4 (50\%) met class Ila indication with the 2011 ACCF/AHA guideline, but none achieved this class of recommendation with the 2014 ESC model.

Conclusion: Low agreement was found between the two strategies. The novel ESC model decreased the ICD recommendations, especially in those with class Ila recommendation, but left unprotected all patients with SCD or appropriate shock. (Arq Bras Cardiol. 2020; 115(2):197-204)

Keywords: Cardiomyopathy, Hypertrophic/genetics; Heredity; Death,Sudden, Cardiac; Arrhythmias, Cardiac; Syncope; Defibrillators, Implantable; Cohort Studies
\end{abstract}

Full texts in English - http://www.arquivosonline.com.br

Correspondência: Beatriz Piva e Mattos •

Hospital de Clínicas de Porto Alegre - Serviço de Cardiologia - Rua Ramiro Barcelos, 2350. CEP 90035-903, Porto Alegre, RS - Brasil

Artigo recebido em 07/03/2019, revisado em 10/07/2019, aceito em 14/08/2019

DOI: https://doi.org/10.36660/abc.20190161 


\section{Introdução}

A cardiomiopatia hipertrófica $(\mathrm{CMH})$ representa a doença cardiovascular de origem genética mais prevalente, com acometimento de um em cada 200 indivíduos. ${ }^{1}$ A morte súbita (MS), atualmente estimada em 0,5 a $1 \%$ ao ano, incide em qualquer faixa etária, embora predomine entre jovens e atletas. ${ }^{2-4}$

A estratificação de risco para MS na $\mathrm{CMH}$ constitui a base para indicação de cardiodesfibrilador implantável (CDI), única modalidade considerada capaz de modificar o prognóstico da doença. ${ }^{4-7}$ Em pacientes com parada cardiorrespiratória prévia a indicação é consensual; contudo, em relação à prevenção primária, permanecem interrogações. Cinco fatores individualizados em estudos longitudinais e validados em metanálise são reconhecidos como preditores independentes de MS: história familiar, síncope inexplicada, espessura parietal máxima do ventrículo esquerdo (EPMVE) $\geq 30 \mathrm{~mm}$, taquicardia ventricular não sustentada (TVNS) e resposta anormal da pressão arterial ao exercício. ${ }^{5-13}$ No consenso American College of Cardiology (ACC)/European Society of Cardiology (ESC) 2003, a indicação de CDI era baseada no número de indicadores de risco. ${ }^{14}$ Os critérios foram atualizados na diretriz American College of Cardiology Foundation (ACCF)/American Heart Association (AHA) 2011 em que foram incorporados fatores modificantes: mutações malignas, realce tardio à ressonância magnética (RM), aneurismas apicais e obstrução da via de saída do VE. ${ }^{15}$ Novo modelo matemático-estatístico, adotado pela ESC em 2014, possibilita, com uma calculadora online, estimar o risco absoluto e a mortalidade em 5 anos por meio da atribuição de pesos aos quatro primeiros preditores independentes mencionados acrescidos por gradiente da via de saída do VE, diâmetro do átrio esquerdo e idade. ${ }^{16,17}$

Em decorrência, o objetivo deste estudo é analisar o impacto do modelo ESC 2014 na determinação do risco de MS e indicação de CDI em prevenção primária em uma coorte de pacientes com $\mathrm{CMH}$ por meio da confrontação com os critérios prévios propostos por ACCF/AHA 2011.

\section{Métodos}

\section{Seleção de pacientes}

Foi analisada, retrospectivamente, uma coorte de 108 indivíduos registrados no ambulatório de $\mathrm{CMH}$ de um hospital universitário de atendimento terciário, entre março de 2007 e março de 2018. Os pacientes foram submetidos a eletrocardiograma em repouso, eletrocardiograma Holter e ecocardiograma, acrescidos por RM ( $n=40 ; 45 \%)$. Estudo genético-molecular foi realizado em 18 (20\%) indivíduos, cujos resultados foram previamente publicados. ${ }^{18}$ O diagnóstico foi estabelecido de acordo com critérios definidos em diretrizes vigentes ${ }^{15,17} \mathrm{com}$ base na identificação de hipertrofia do VE detectada ao ecocardiograma e/ou RM pela presença de EPMVE $\geq 15 \mathrm{~mm}$ medida em qualquer segmento, com razão septo/parede posterior $\geq 1,3$ na ausência de dilatação da câmara e outras condições capazes de produzir alterações semelhantes. Foram excluídos 18 casos por apresentarem período de seguimento $<12$ meses ou história prévia de parada cardiorrespiratória, fibrilação ventricular ou taquicardia ventricular com repercussão hemodinâmica. Os seguintes desfechos foram considerados para análise: 1) MS: fibrilação ventricular documentada, óbito até 1 hora do início de novos sintomas ou durante a noite sem agravamento clínico prévio; 2) choque apropriado pelo CDI por taquicardia ventricular ou fibrilação ventricular. O estudo foi aprovado pelo Comitê de Ética da instituição e desenvolvido de acordo com os princípios da Declaração de Helsinki. Termo de consentimento livre e esclarecido foi obtido de todos os pacientes.

\section{Estratificação de risco para morte súbita}

Os seguintes preditores foram pesquisados: 1) idade; 2) história familiar de MS em primeiro grau, em $<40$ anos de idade ou em qualquer idade com diagnóstico confirmado da doença; 3) EPMVE medida ao ecocardiograma bidimensional; 4) síncope inexplicada nos últimos 6 meses; 5) TVNS definida por três ou mais batimentos extrassistólicos ventriculares sucessivos com frequência cardíaca $\geq 120$ batimentos e duração <30s; 6) resposta anormal da pressão arterial ao exercício expressa pela incapacidade de elevar a pressão arterial sistólica $\geq 25 \mathrm{mmHg}$ ou queda $\geq 10 \mathrm{mmHg}$ no pico do exercício; 7) diâmetro do átrio esquerdo determinado ao ecocardiograma uni ou bidimensional; 8) gradiente máximo na via de saída do VE em repouso ou sob manobra de Valsalva com Doppler contínuo. Os seguintes modificantes de risco foram valorizados: 1) obstrução da via de saída do $\mathrm{VE} \geq 30 \mathrm{mmHg}$; 2) realce tardio com gadolínio à RM cardíaca; 3) aneurisma apical do VE; 4) mutação genética maligna.

O escore ESC HCM-Risk-SCD para estimativa de risco de MS em 5 anos foi calculado pela seguinte fórmula:

Probabilidade em 5 anos $=1-0,998$ exp (índice prognóstico)

Índice prognóstico $=[0,15939858 \times \operatorname{EPMVE}(\mathrm{mm})]-$ $\left[0,00294271 \times \operatorname{EPMVE}^{2}\left(\mathrm{~mm}^{2}\right)\right]+[0,0259082 \times$ diâmetro do átrio esquerdo $(\mathrm{mm})]+[0,00446131 \times$ gradiente máximo na via de saída do VE (repouso/Valsalva)(mmHg)] + [0,4583082 $\times$ história familiar de MS $]+[0,82639195 \times$ TVNS $]+$ [0,71650361 X síncope inexplicada $]-[0,01799934 \times$ idade na avaliação(anos)].

\section{Critérios de indicação de cardiodesfibrilador automático implantável}

Os seguintes critérios para indicação de CDI em prevenção primária foram comparados:

1. Diretriz ACCF/AHA 2011: Classe Ila - história familiar de MS em $1^{\circ}$ grau ou espessura parietal máxima do VE $\geq 30$ mm ou síncope inexplicada recente; Classe Ila - TVNS ou resposta anormal da pressão arterial ao exercício associada a outros fatores ou modificantes de risco; Classe IIb - TVNS ou resposta anormal da pressão arterial ao exercício isoladas; Classe III - ausência dos fatores anteriormente citados.

2. Diretriz ESC 2014: Classe Ila - HCM-Risk-SCD $\geq 6 \%$; Classe IIb $-<6 \%$ e $\geq 4 \%$; Classe III $-<4 \%$.

\section{Análise estatística}

Os dados quantitativos foram apresentados por meio de média e desvio-padrão para distribuição normal ou por 
meio de medianas e intervalos interquartílicos (percentis 25 e 75) para distribuição não normal. A normalidade dos dados foi testada pelo teste Shapiro-Wilk. As variáveis categóricas foram descritas por meio de frequências absolutas e relativas. Variáveis contínuas com distribuição simétrica foram aferidas pelo teste $t$ de Student para amostras independentes e análise de variâncias (ANOVA One-Way), variáveis categóricas pelos testes qui-quadrado e exato de Fisher e diferenças entre as categorias por meio dos resíduos ajustados. O coeficiente Kappa foi determinado para analisar a concordância entre as indicações para implante de CDI entre as diretrizes ACCF/AHA 2011 e ESC 2014. Os percentuais atingidos por cada um dos preditores que compõe o escore ESC HCM-Risk-SCD foram calculados pela média ponderada da variação de cada preditor na equação sobre a soma das variações desses preditores. A estimativa de sobrevida da amostra foi determinada por meio de curva de Kaplan-Meier. O tamanho da amostra foi estimado em 70 indivíduos para um valor esperado de Kappa $=0,3$, se considerada a ocorrência de concordância entre as sistematizações, Kappa $=0$, para poder de 90\% e $p<0,05$. Os dados foram processados no software SPSS versão 20.0 (SPSS Inc., Chicago, Illinois, EUA). O nível de significância adotado nas análises estatísticas foi de 5\%.

\section{Resultados}

\section{Características clínicas}

A população em estudo foi constituída por 90 pacientes consecutivos com $\mathrm{CMH}$, com idade média de $62 \pm 12$ anos, $85(94 \%) \geq 40$ anos e 56 (62\%) do sexo feminino. As características clínicas da amostra encontram-se na Tabela 1. No período médio de seguimento de $6 \pm 3$ anos, $15(17 \%)$ implantaram CDI para prevenção primária de MS. Dois (2\%) pacientes apresentaram choque apropriado, $6(7 \%)$ MS e $6(7 \%)$ morte por outras causas (Tabela 2$)$.

A sobrevida livre de MS ou choque apropriado pelo CDI no período de seguimento médio de 5 anos foi de $93 \%$ e, em 10 anos, de $92 \%$. A sobrevida livre de morte por todas as causas em 5 e 10 anos foi de $80 \%$.

\section{Estratificação de risco para morte súbita pelo escore ESC HCM-risk SCD}

O escore ESC HCM-risk-SCD foi, em média, de 3,2 $\pm 2,5 \%$ na amostra, estimado como baixo ( $<4 \%)$ em $67(75 \%)$, médio $(\geq 4 \%$ e $<6 \%)$ em 11 (12\%) e alto (>6\%) em $12(13 \%)$. A análise comparativa dos indicadores de MS valorizados nas duas normativas entre as três faixas de risco identificou que TVNS [3 (4\%) vs. $6(54 \%)$ vs. $8(67 \%), p=0,0001]$, síncope [6 (9\%) vs. $3(27 \%)$ vs. $7(58 \%), p=0,0001]$ e EPMVE mais elevada $(17 \pm 3 \mathrm{~mm}$ vs. $21 \pm 2 \mathrm{~mm}$ vs. $21 \pm 8 \mathrm{~mm}, \mathrm{p}=0,002)$ foram predominantes entre aqueles com maior predisposição. Os demais preditores não diferiram entre os grupos (Tabela 3). Choque apropriado pelo CDI ou MS foi semelhante entre os pacientes das faixas de baixo, médio e alto risco $[6(8,8 \%)$ vs. $2(18,2 \%)$ vs. $0(0 \%), p=0,22]$.

A Tabela 4 apresenta os percentuais atingidos por cada um dos preditores que compõe o escore ESC HCM-Risk-SCD
Tabela 1 - Características clínicas de 90 pacientes com cardiomiopatia hipertrófica

\begin{tabular}{|c|c|}
\hline Idade (anos) & $62 \pm 12$ \\
\hline Idade >40 anos $(n, \%)$ & $85(94 \%)$ \\
\hline Sexo feminino $(n, \%)$ & $56(62 \%)$ \\
\hline \multicolumn{2}{|l|}{ Classe funcional NYHA } \\
\hline$I / I I(n, \%)$ & $75(83 \%)$ \\
\hline III/IV $(n, \%)$ & $15(17 \%)$ \\
\hline Cardiopatia isquêmica $(\mathrm{n}, \%)$ & $11(12 \%)$ \\
\hline \multicolumn{2}{|l|}{ Terapêutica } \\
\hline Betabloqueadores $(n, \%)$ & $70(78 \%)$ \\
\hline Amiodarona $(n, \%)$ & $20(22 \%)$ \\
\hline Verapamil/diltiazem $(n, \%)$ & $24(27 \%)$ \\
\hline \multicolumn{2}{|l|}{ Ecocardiograma } \\
\hline Diâmetro do AE (mm) & $44 \pm 7$ \\
\hline Diâmetro diastólico do VE (mm) & $43 \pm 6$ \\
\hline Diâmetro sistólico do VE (mm) & $34 \pm 5$ \\
\hline Espessura diastólica do septo (mm) & $19 \pm 4$ \\
\hline Espessura diastólica da parede posterior do VE (mm) & $11 \pm 2$ \\
\hline Fração de ejeção (\%) & $71 \pm 9$ \\
\hline $\mathrm{E} / \mathrm{E}^{\prime}$ & $16 \pm 8$ \\
\hline Gradiente na via de saída do VE em repouso (mmHg) & $28 \pm 31$ \\
\hline Gradiente na via de saída do VE sob Valsalva ( $\mathrm{mmHg}$ ) & $36 \pm 38$ \\
\hline \multicolumn{2}{|l|}{ Fatores de risco para MS } \\
\hline História familiar de MS* & $23(26 \%)$ \\
\hline TVNS $^{*}$ & $17(19 \%)$ \\
\hline Síncope ${ }^{*}$ & $16(18 \%)$ \\
\hline Resposta anormal da PA ao exercício* & $9(10 \%)$ \\
\hline EPMVE $>30 \mathrm{~mm}^{*}$ & $1(1 \%)$ \\
\hline Obstrução VSVE $\geq 30 \mathrm{mmHg}^{\dagger}$ & $44(49 \%)$ \\
\hline Realce tardio à RM $†$ & $11(12 \%)$ \\
\hline Aneurisma apical de $\mathrm{VE}^{\dagger}$ & 0 \\
\hline Mutação maligna $^{\dagger}$ & 0 \\
\hline \multicolumn{2}{|l|}{ Número de fatores de risco } \\
\hline 0 & $42(47 \%)$ \\
\hline 1 & $32(35 \%)$ \\
\hline$\geq 2$ & $16(18 \%)$ \\
\hline $\begin{array}{l}\text { *Preditores independentes; }{ }^{\dagger} \text { Fatores modificantes; } N Y \\
\text { Association; AE: átrio esquerdo; VE: ventrículo e } \\
\text { súbita; TVNS: taquicardia ventricular não sustentada } \\
\text { parietal máxima do ventrículo esquerdo; PA: pressão } \\
\text { saída do ventrículo esquerdo; RM: ressonância magr }\end{array}$ & $\begin{array}{l}\text { Ork Heart } \\
\text { S: morte } \\
\text { spessura } \\
\text { IE: via de }\end{array}$ \\
\hline
\end{tabular}

nas três categorias de risco para MS. Os fatores que mais contribuíram para o cálculo do escore nas faixas de baixo, médio e alto risco foram EPMVE, diâmetro do átrio esquerdo e idade. Gradiente na via de saída do VE, história familiar de MS, TVNS e síncope evidenciaram menor contribuição. 


\section{Artigo Original}

Comparação das diretrizes American College of Cardiology Foundation/American Heart Association 2011 e European Society of Cardiology 2014

De acordo com os critérios ACCF/AHA 2011, 43 (48\%) pacientes receberam indicação de CDI Classe Ila, três (3\%) Classe IIb e 44 (49\%) Classe III. Com base na diretriz ESC 2014, 12 (14\%) pacientes constituíram recomendação Classe IIa, 11 (12\%) Classe IIb e 67 (74\%) Classe III. A comparação dos graus de indicação de CDI entre as duas sistematizações revelou baixa concordância (Kappa $=0,355$, $\mathrm{p}=0,0001)$. O escore ESC HCM-risk-SCD reduziu a classe de recomendação de CDI em 32 (36\%) pacientes, manteve em 57 (63\%) e acrescentou status para implante em somente um (1\%). Dos 43 (48\%) indivíduos que receberam indicação Ila pela ACCF/AHA 2011, 32 (74\%) tiveram a recomendação
Tabela 2 - Dados evolutivos de 90 pacientes com cardiomiopatia hipertrófica seguidos por $6 \pm 3$ anos

\begin{tabular}{lc}
\hline Insuficiência cardiaca Classe III/IV & $20(22 \%)$ \\
Fibrilação atrial (n,\%) & $29(32 \%)$ \\
Ablação alcoólica do septo (n,\%) & $9(10 \%)$ \\
Miectomia cirúrgica $(n, \%)$ & $3(3 \%)$ \\
Marca-passo (n,\%) & $6(7 \%)$ \\
Implante de CDI (n,\%) & $15(17 \%)$ \\
Choque apropriado CDI (n,\%) & $2(2 \%)$ \\
Morte súbita (n,\%) & $6(7 \%)$ \\
Morte por outras causas (n,\%) & $6(7 \%)$ \\
\hline
\end{tabular}

CDI: cardiodesfibrilador implantável.

Tabela 3 - Distribuição dos preditores de morte súbita nas três faixas de risco da diretriz European Society of Cardiology 2014

\begin{tabular}{|c|c|c|c|c|}
\hline & \multicolumn{3}{|c|}{ Risco de MS } & \multirow[b]{2}{*}{$\mathrm{p}$} \\
\hline & $\begin{array}{c}<4 \% \\
(n=67 ; 75 \%)\end{array}$ & $\begin{array}{c}\geq 4 \%-<6 \\
(n=11 ; 12 \%)\end{array}$ & $\begin{array}{c}\geq 6 \% \\
(n=12 ; 13 \%)\end{array}$ & \\
\hline Idade (anos) & $64 \pm 11$ & $60 \pm 17$ & $57 \pm 13$ & 0,156 \\
\hline História familiar de MS & $14(21 \%)$ & $4(36 \%)$ & $5(42 \%)$ & 0,177 \\
\hline Síncope & $6(9 \%)$ & $3(27 \%)$ & $7(58 \%)$ & 0,0001 \\
\hline EPMVE $\geq 30 \mathrm{~mm}$ & 0 & 0 & 1 & 0,264 \\
\hline TVNS & $3(4 \%)$ & $6(54 \%)$ & $8(67 \%)$ & 0,0001 \\
\hline Resposta anormal da PA ao exercício & $8(12 \%)$ & 0 & $1(8 \%)$ & 0,595 \\
\hline Realce tardio à ressonância magnética & $8(12 \%)$ & $1(9 \%)$ & $2(17 \%)$ & 0,822 \\
\hline Obstrução VSVE $\geq 30 \mathrm{mmHg}$ & $31(46 \%)$ & $7(64 \%)$ & $6(50 \%)$ & 0,649 \\
\hline Diâmetro do átrio esquerdo (mm) & $46 \pm 7$ & $48 \pm 9$ & $48 \pm 8$ & 0,545 \\
\hline Espessura parietal máxima do VE (mm) & $17 \pm 3$ & $21 \pm 2$ & $21 \pm 8$ & 0,002 \\
\hline Gradiente máximo VSVE (mmHg) & $33 \pm 42$ & $45 \pm 39$ & $40 \pm 44$ & 0,77 \\
\hline
\end{tabular}

MS: morte súbita; EPMVE: espessura parietal máxima do ventrículo esquerdo; TVNS: taquicardia ventricular não sustentada; PA: pressão arterial; VSVE: via de saída do ventrículo esquerdo; VE: ventrículo esquerdo.

Tabela 4 - Contribuição dos preditores de morte súbita para o cálculo do escore ESC HCM-Risk-SCD

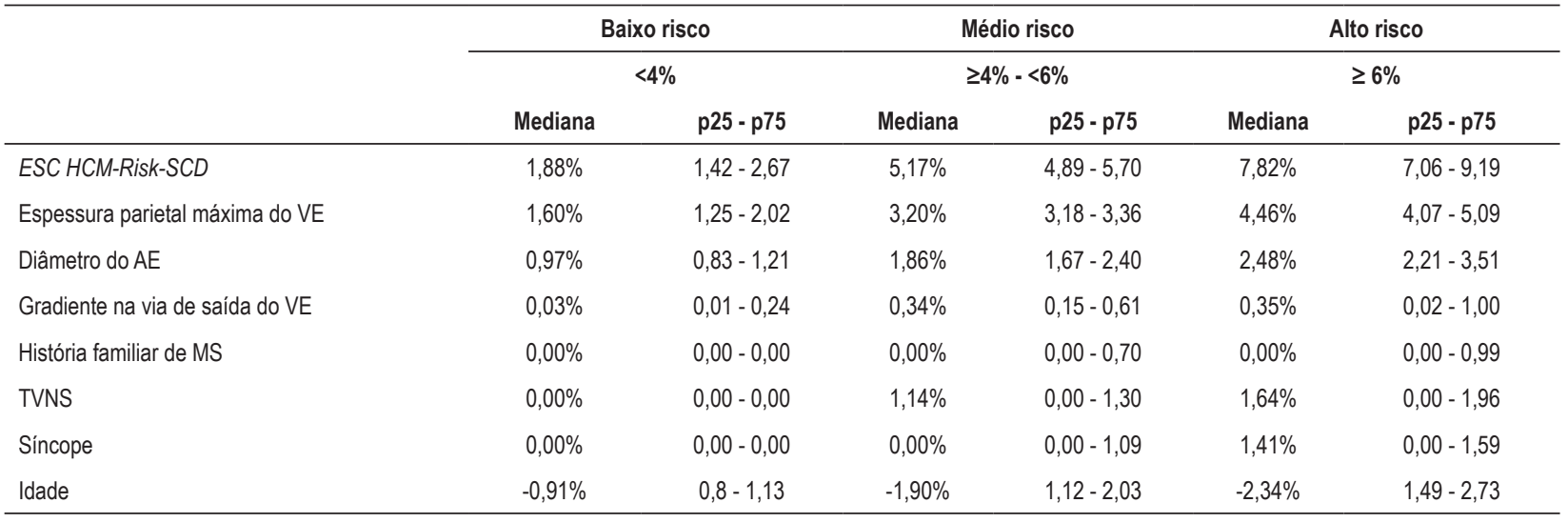

ESC: European Society of Cardiology; VE: ventrículo esquerdo; AE: átrio esquerdo; MS: morte súbita; TVNS: taquicardia ventricular não sustentada. 
reduzida pela ESC 2014, 8 (18\%) para Ilb e 24 (56\%) para III. Apenas 11(26\%) conservaram a indicação Classe Ila. Dos 44 pacientes (49\%) em Classe III pela ACCF/AHA 2011, 43 (98\%) mantiveram a contraindicação pela sistematização europeia (Tabela 5). A Figura 1 apresenta o resumo do estudo e seus principais resultados.

O escore ESC foi de $3 \pm 1,7 \%$ nos 8 (9\%) pacientes que sofreram MS ou choque apropriado. Quatro (50\%) apresentavam indicação Classe Ila para implante do dispositivo pela ACCF/AHA 2011, mas nenhum atingiu esse grau de recomendação pela ESC 2014, ainda que 2 (25\%) pacientes tenham permanecido em Classe IIb.

Os fatores de risco que na amostra caracterizaram recomendação Ila pela ACCF/AHA 2011 conjuntamente associaram-se à perda de indicação na reestratificação pela ESC $(p=0,05)$. História familiar de MS e TVNS adicionada à obstrução da via de saída de VE foram os preditores que apresentaram maior perda de recomendação de CDI pela diretriz europeia (Tabela 6).

\section{Discussão}

Neste estudo, confrontamos, pela primeira vez, os critérios para prevenção primária de MS propostos pela ACCF/AHA 2011 e ESC 2014 em uma população brasileira de portadores de $\mathrm{CMH}$ com base em uma coorte de pacientes ambulatoriais não referenciados. Nossos resultados demonstram baixa concordância entre as duas sistematizações quanto aos critérios de indicação de CDI em prevenção primária. O escore ESC HCM-Risk-SCD reduziu a classe de recomendação para implante em relação a ACCF/AHA em 36\% dos pacientes. Entre aqueles situados em Classe Ila pela diretriz norte-americana, $74 \%$ tiveram o grau de indicação reduzido pela ESC e 56\% configuraram contraindicação para implante de CDI, sendo a recomendação mantida em apenas 26\%. O escore europeu acrescentou indicação em somente $1 \%$ dos pacientes. Na quase totalidade dos casos em que o implante não foi recomendado pela diretriz norte-americana, a contraindicação foi mantida pelos critérios da ESC. O novo modelo excluiria da Classe Ila os 8 (9\%) pacientes que sofreram MS ou choque apropriado pelo CDI durante o período de observação, ainda que $25 \%$ desses tenham permanecido em Classe Ilb.

A $\mathrm{CMH}$ é uma cardiopatia de caráter arritmogênico, cujo substrato histopatológico constituído por hipertrofia, desorganização celular, fibrose e doença da microcirculação favorece o desenvolvimento de arritmias ventriculares fatais. 5,6,19,20 A estratificação de risco para MS baseia-se em dados observacionais coletados de populações muito selecionadas. É considerada complexa em decorrência do caráter heterogêneo da doença e julgada ainda imperfeita pelo fato de muitos óbitos ocorrerem na ausência de preditores. ${ }^{5-7,21} \mathrm{~A}$ limitação oferecida pelos algoritmos de 2003 e 2011 foi demonstrada em um registro internacional que evidenciou ausência de diferença nas taxas de choques apropriados entre os pacientes com um, dois, três ou mais predisponentes. ${ }^{22}$ Análise posterior de validação desses critérios verificou que a incidência de MS e choque apropriado não divergiu entre pacientes com um ou nenhum preditor e que os algoritmos iniciais teriam poder limitado para discriminar indivíduos com maior ou menor predisposição e implicariam em número substancial de implantes desnecessários. ${ }^{23}$

No presente estudo, foi avaliada uma coorte de $\mathrm{CMH}$ com faixa etária mais avançada e características de baixo risco: 78\% mantiveram-se em classe funcional I/II, 47\% não apresentavam fatores predisponentes para MS e 35\% evidenciavam apenas um. Portadores de $\mathrm{CMH} \geq 60$ anos de idade exibem taxas mais reduzidas de morbimortalidade e MS, mesmo na presença de preditores. ${ }^{6} \mathrm{~A}$ sobrevida livre de MS ou choque apropriado pelo CDI atingiu 93\% em 5 anos e 92\% em 10 anos, sendo que, no período, apenas $9 \%$ dos indivíduos desenvolveram esses eventos. Estudo longitudinal, multicêntrico, demonstra resultados semelhantes e destaca que a $\mathrm{CMH}$, quando convenientemente tratada, apresenta reduzida mortalidade na fase adulta, com sobrevida média em 10 anos semelhante à esperada na população em geral. ${ }^{3}$

O escore ESC HCM-risk-SCD, de 3,2 $22,5 \%$, caracterizou risco reduzido de MS em 75\% dos pacientes. TVNS, síncope e maior EPMVE foram mais frequentes na faixa de alto risco em relação às demais. Nesse estudo, determinamos os percentuais atingidos por cada um dos preditores integrantes do escore nas três categorias de risco com objetivo de discriminar aqueles que atingiram maior peso e que pudessem justificar a baixa concordância entre as duas diretrizes. Verificou-se que os fatores que mais contribuíram para o cálculo, por terem alcançado valores mais elevados nas faixas de baixo, médio e alto risco, foram EPMVE, diâmetro do átrio esquerdo e idade, a qual tem efeito subtrativo. Esses achados podem justificar a baixa concordância entre as duas diretrizes, visto que EPMVE como variável contínua, diâmetro do átrio esquerdo e idade não constam da normativa norteamericana. História familiar de MS e síncope isoladas, que na

Tabela 5 - Comparação das indicações de cardiodesfibrilador implantável entre as diretrizes American College of Cardiology Foundation/ American Heart Association 2011 e European Society of Cardiology 2014

\begin{tabular}{|c|c|c|c|c|c|}
\hline & & \multirow[b]{3}{*}{$\mathrm{n}(\%)$} & \multicolumn{3}{|c|}{ ESC 2014} \\
\hline & & & lla & llb & III \\
\hline & & & $12(14 \%)$ & $11(12 \%)$ & $67(74 \%)$ \\
\hline & Ila & $43(48 \%)$ & $11(26 \%)$ & $8(18 \%)$ & $24(56 \%)$ \\
\hline \multirow[t]{3}{*}{ ACCF/AHA 2011} & $\| l b$ & $3(3 \%)$ & 0 & $3(100 \%)$ & 0 \\
\hline & III & $44(49 \%)$ & $1(2 \%)$ & 0 & $43(98 \%)$ \\
\hline & & \multicolumn{4}{|c|}{ Kappa $=0,355, P=0,0001$} \\
\hline
\end{tabular}




\section{Artigo Original}

\section{Implante de CDI em prevenção primária de morte súbita 90 pacientes com $\mathrm{CMH}$}

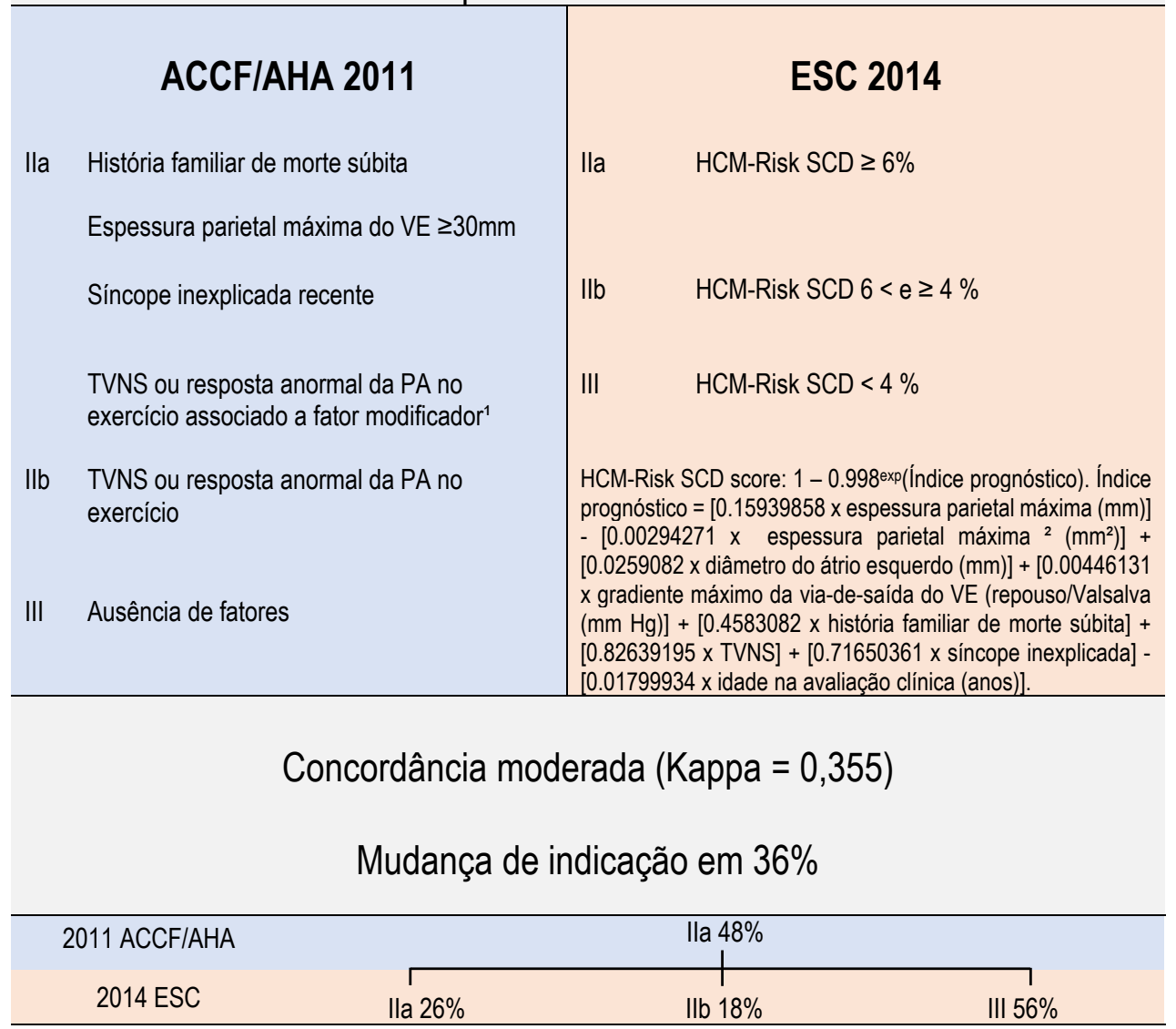

Figura 1 - Discordância entre diretrizes ACCF/AHA 2011 e ESC 2014 sobre critérios de prevenção primária de morte súbita na cardiomiopatia hipertrófica

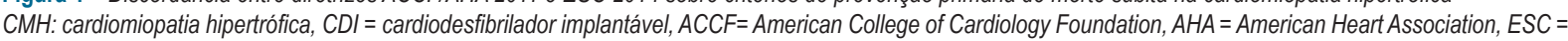
European Society of Cardiology, VE=ventrículo esquerdo, TVNS, taquicardia ventricular não-sustentada, $P A=$ pressão arterial. 'Fator modificador: 1. Obstrução da via de saída do VE $\geq 30 \mathrm{mmHg}$; 2. Realce tardio com gadolíneo à ressonância magnética cardíaca; 3. Aneurisma apical do VE; 4. Mutação genética maligna.

Tabela 6 - Perfil de risco para morte súbita em pacientes com cardiomiopatia hipertrófica e indicação Classe lla de cardiodesfibrilador implantável pela American College of Cardiology Foundation/American Heart Association 2011: reestratificação de risco pela European Society of Cardiology 2014

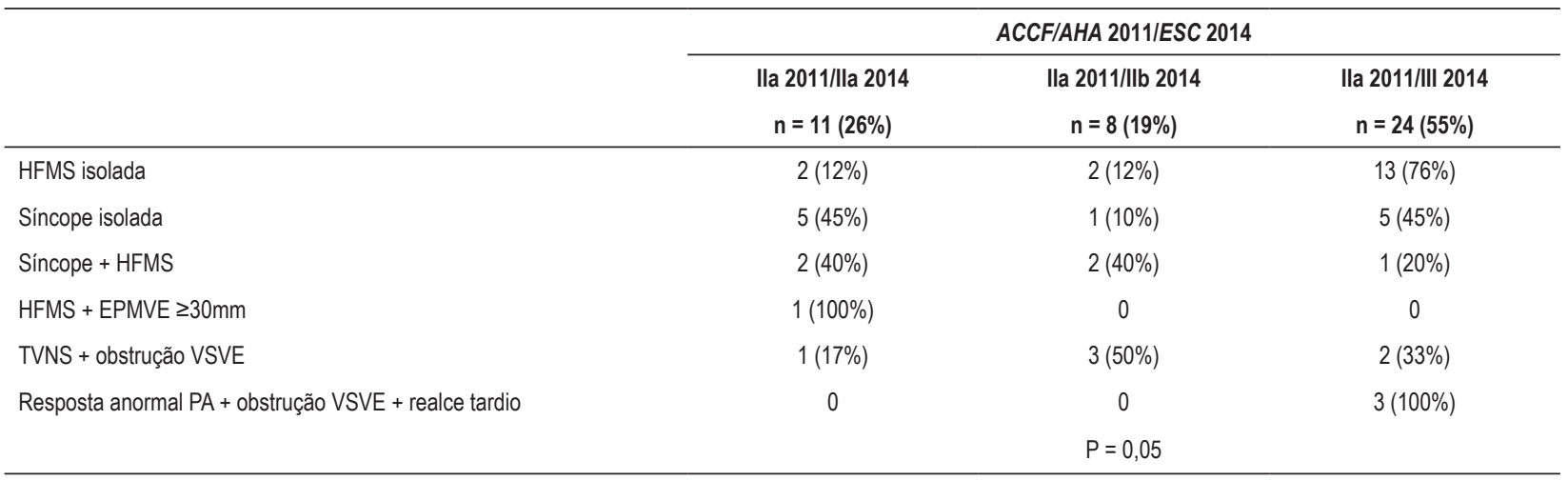

HFMS: história familiar de morte súbita; EPMVE: espessura parietal máxima do ventrículo esquerdo; TVNS: taquicardia ventricular não sustentada; VSVE: via de saída do ventrículo esquerdo; PA: pressão arterial; ACCF: American College of Cardiology Foundation. AHA: American Heart Association; ESC: European Society of Cardiology. 
ACCF/AHA constituem indicação para implante, evidenciaram menor contribuição para o cálculo do escore.

Os fatores de risco que na amostra caracterizaram indicação Ila para $C D I$ pela $A C C F / A H A$, notadamente, história familiar de MS e TVNS adicionada à obstrução da via de saída do VE, associaram-se à perda de recomendação pela ESC, atingindo status de contraindicação em 55\% dos casos. Nossos resultados sugerem que a perda de recomendação para implante de CDI proporcionada pelo escore europeu se relaciona sobretudo a situações em que a indicação pela diretriz norte-americana está fundamentada na presença de um preditor isolado ou em associação a fatores modificantes. Esses achados são justificados pelo fato de que o modelo europeu define prevenção primária com base no conjunto de fatores de risco e não na presença de um só indicador.

O escore ESC HCM-Risk-SCD foi validado de modo independente em populações de três continentes em estudos observacionais que, em sua maioria, demonstram que o novo modelo contribui para o aprimoramento da estratificação de risco e do processo decisório na $\mathrm{CMH} .{ }^{24-28}$ Outras análises assinalam a pouca sensibilidade do escore para o reconhecimento dos pacientes mais suscetíveis, a maior capacidade de identificar casos sem indicação de CDI e o registro de taxas similares de eventos nas três faixas de risco. ${ }^{29-32}$ Nosso estudo corrobora esses achados ao demonstrar que o modelo europeu reduz as recomendações de $\mathrm{CDI}$ em relação à diretriz norte-americana, deixa desprotegida a totalidade de pacientes que sofreram MS ou choque apropriado e estabelece maior concordância nos casos de contraindicação de implante do dispositivo. Contudo, a metanálise de seis estudos, com 7.291 pacientes, demonstra que, na maioria dos casos, a suscetibilidade à MS em 5 anos foi adequadamente estimada pelo escore ESC. ${ }^{33}$

O escore europeu fundamenta a estratificação de risco para MS por meio da aplicação de um rígido modelo matemáticoestatístico em uma doença complexa de evolução imprevisível. Limitações metodológicas podem existir na dependência da avaliação do átrio esquerdo pelo respectivo diâmetro, da obstrução da via de saída pela manobra de Valsalva e pela exclusão de isquemia miocárdica, realce tardio e aneurismas apicais do VE. Embora restrições possam ser admitidas em relação ao seu desempenho, particularmente no alto risco, o escore europeu merece assimilação na prática clínica como forma validada de orientar decisões terapêuticas. A determinação dos percentuais atingidos pelos preditores integrantes da fórmula caso a caso poderá contribuir para a interpretação de resultados na prática clínica. Na presente análise, a normativa norte-americana asseguraria proteção a um número maior de indivíduos em relação ao critério europeu. Todavia, poderia resultar em implantes desnecessários e exporia essa população a complicações inerentes ao dispositivo, tais como infecções e choques inapropriados. ${ }^{4,15,17}$
São necessários estudos prospectivos validados igualmente em populações de menor risco, com o objetivo de possibilitar a identificação de novos fatores predisponentes e o refinamento dos critérios de indicação de CDI para prevenção primária de MS nessa doença.

\section{Limitações do estudo}

O presente estudo baseia-se na avaliação de uma coorte bem documentada de $\mathrm{CMH}$ de um único centro com menor grau de seleção e faixa etária mais avançada. As características clínicas e as reduzidas taxas de eventos indicam tratar-se de pacientes de menor risco, distintos daqueles que integram a maioria dos estudos de validação. Esses aspectos podem limitar as conclusões a populações com esse perfil. Contudo, os casos incluídos são tão representativos da doença quanto aqueles de maior risco e mais suscetíveis a complicações, selecionados em centros de referência.

\section{Conclusão}

No estudo de uma coorte de $\mathrm{CMH}$ de menor risco e faixa etária mais avançada, baixa concordância foi identificada entre os critérios de prevenção primária de MS estabelecidos pelas diretrizes ACCF/AHA 2011 e ESC 2014. O escore ESC HCM-Risk$S C D$ reduziu as indicações para implante de CDI na população estudada, notadamente naqueles situados em Classe Ila na sistematização norte-americana, mas deixou desprotegida a totalidade de pacientes que sofreram MS ou choque apropriado. A maior contribuição para o cálculo do escore de preditores não incluídos na normativa ACCF/AHA 2011 poderia justificar, em parte, a discordância entre as duas diretrizes.

\section{Contribuição dos autores}

Concepção e desenho da pesquisa, Obtenção de dados, Análise e interpretação dos dados e Redação do manuscrito: Mattos BP, Scolari FL e Garbin HI; Análise estatística e Revisão crítica do manuscrito quanto ao conteúdo intelectual importante: Mattos BP, Scolari FL.

\section{Potencial conflito de interesses}

Declaro não haver conflito de interesses pertinentes.

\section{Fontes de financiamento}

O presente estudo não teve fontes de financiamento externas.

\section{Vinculação acadêmica}

Não há vinculação deste estudo a programas de pósgraduação.

\section{Referências}

1. Semsarian C, Ingles J, Maron MS, Maron BJ. New perspectives on the prevalence of hypertrophic cardiomyopathy. J Am Coll Cardiol. 2015;65(12):1249-54.

2. Mattos BP. Sudden death risk stratification in hypertrophic cardiomyopathy: genetic and clinical bases. Arq Bras Cardiol. 2006;87(3):391-9.
3. Maron BJ, Rowin EJ, Casey SA, Link MS, Lesser JR, Chan RH, et al. Hypertrophic cardiomyopathy in adulthood associated with low cardiovascular mortality with contemporary management strategies. J Am Coll Cardiol. 2015;65(18):1915-28.

4. Maron BJ. Clinical course and management of hypertrophic cardiomyopathy. $\mathrm{N}$ Engl J Med. 2018;379(7):655-68. 
5. O'Mahony C, Elliott PM. Prevention of sudden cardiac death in hypertrophic cardiomyopathy. Heart. 2014;100(3):254-60.

6. Maron BJ, Maron MS. Contemporary strategies for risk stratification and prevention of sudden death with the implantable defibrillator in hypertrophic cardiomyopathy. Heart Rhythm. 2016;13(5):1155-65.

7. Weissler-Snir A, Adler A, Williams L, Gruner C, Rakowski H. Prevention of sudden death in hypertrophic cardiomyopathy: bridging the gaps in knowledge. Eur Heart J. 2017;38(22):1728-37.

8. Elliott PM, Poloniecki J, Dickie S, Sharma S, Monserrat L, Varnava A, et al. Sudden death in hypertrophic cardiomyopathy: identification of high risk patients. J Am Coll Cardiol. 2000;36(7):2212-8.

9. Spirito P, Bellone P, Harris KM, Bernabo P, Bruzzi P, Maron BJ. Magnitude of left ventricular hypertrophy and risk of sudden death in hypertrophic cardiomyopathy. N Engl J Med. 2000;342(24):1778-85.

10. Monserrat L, Elliott PM, Gimeno JR, Sharma S, Penas-Lado M, McKenna WJ. Non-sustained ventricular tachycardia in hypertrophic cardiomyopathy: an independent marker of sudden death risk in young patients. J Am Coll Cardiol. 2003;42(5):873-9.

11. Elliott PM, Gimeno JR, Tomé MT, Shah J, Ward D, Thaman R, et al. Left ventricular outflow tract obstruction and sudden death risk in patients with hypertrophic cardiomyopathy. Eur Heart J. 2006;27(16):1933-41.

12. Spirito P, Autore C, Rapezzi C, Bernabò P, Badagliacca R, Maron MS, et al. Syncope and risk of sudden death in hypertrophic cardiomyopathy. Circulation. 2009;119(13):1703-10.

13. Christiaans I, van Engelen K, van Langen IM, Birnie E, Bonsel GJ, Elliott PM, et al. Risk stratification for sudden cardiac death in hypertrophic cardiomyopathy: systematic review of clinical risk markers. Europace. 2010;12(3):313-21.

14. Maron BJ, McKenna WJ, Danielson GK, Kappenberger LJ, Kuhn HJ, Seidman CE, et. al. American College of Cardiology/European Society of Cardiology clinical expert consensus document on hypertrophic cardiomyopathy. A report of the American College of Cardiology Foundation task force on clinical expert consensus documents and the European Society of Cardiology Committee for practice guidelines. J Am Coll Cardiol. 2003;42(9):1687-713.

15. Gersh BJ, Maron BJ, Bonow RO, Dearani JA, Fifer MA, Link MS, et al. 2011 ACCF/AHA guideline for the diagnosis and treatment of hypertrophic cardiomyopathy: executive summary: a report of the American College of Cardiology Foundation/American Heart Association Task Force On Practice Guidelines. Circulation. 2011;124(24):2761-96.

16. O'Mahony C, Jichi F, Pavlou M, Monserrat L4, Anastasakis A5, Rapezzi $\mathrm{C}$, et al. A novel clinical risk prediction model for sudden cardiac death in hypertrophic cardiomyopathy (HCM risk-SCD). Eur Heart J. 2014;35(30):2010-20.

17. Authors/Task Force members, Elliott PM, Anastasakis A, Borger MA, Borggrefe M, Cecchi F, et al. 2014 ESC Guidelines on diagnosis and management of hypertrophic cardiomyopathy: the task force for the diagnosis and management of hypertrophic cardiomyopathy of the European Society of Cardiology (ESC). Eur Heart J. 2014;35(39):2733-79.

18. Mattos BP, Scolari FL, Torres MAR, Simon L, Freitas VC, Giugliani $\mathrm{R}$, et al. Prevalence and phenotypic expression of mutations in the MYH7, MYBPC3 and TNNT2 genes in families with hypertrophic cardiomyopathy in the south of Brazil: a cross-sectional study. Arq Bras Cardiol. 2016;107(3):257-65.

19. Shiozaki AA, Senra T, Arteaga E, Pita CG, Martinelli Filho M, Ávila LFR, et al. Myocardial fibrosis in patients with hypertrophic cardiomyopathy and high risk for sudden death. Arq Bras Cardiol. 2010;94(4):535-40.
20. Mattos BP, Torres MAR, Freitas VC, Scolari FL, Loreto MS. Ventricular arrhythmias and left ventricular hypertrophy in hypertrophic cardiomyopathy. Arq Bras Cardiol. 2013;100(5):452-9.

21. Spirito P, Autore C, Formisano F, Assenza GE, Biagini E, Haas TS, et al. Risk of sudden death and outcome in patients with hypertrophic cardiomyopathy with benign presentation and without risk factors. Am J Cardiol. 2014;113(9):1550-5.

22. Maron BJ, Spirito P, Shen WK, Haas TS, Formisano F, Link MS, et. al. Implantable cardioverter-defibrillators and prevention of sudden cardiac death in hypertrophic cardiomyopathy. JAMA. 2007;298(4):405-12.

23. O'Mahony C, Tome-Esteban M, Lambiase PD, Pantazis A, Dickie S, McKenna WJ, et al. A validation study of the 2003 American College of Cardiology/European Society of Cardiology and 2011 American College of Cardiology Foundation/American Heart Association risk stratification and treatment algorithms for sudden cardiac death in patients with hypertrophic cardiomyopathy. Heart. 2013;99(8):534-41.

24. Vriesendorp PA, Schinkel AF, Liebregts $M$, Theuns DA, van Cleemput J, Ten Cate FJ, et al. Validation of the 2014 European Society of Cardiology guidelines risk prediction model for the primary prevention of sudden cardiac death in hypertrophic cardiomyopathy. Circ Arrhythm Electrophysiol. 2015;8(4):829-35

25. Ruiz-Salas A, García-Pinilla JM, Cabrera-Bueno F, Fernández-Pastor J, Peña-Hernández J, Medina-Palomo C, et al. Comparison of the new risk prediction model (HCM Risk-SCD) and classic risk factors for sudden death in patients with hypertrophic cardiomyopathy and defibrillator. Europace. 2016;18(5):773-7.

26. Fernández A, Quiroga A, Ochoa JP, Mysuta M, Casabé JH, Biagetti M, et al. Validation of the 2014 European Society of Cardiology sudden cardiac death risk prediction model in hypertrophic cardiomyopathy in a reference center in South America. Am J Cardiol. 2016;118(1):121-6.

27. O'Mahony C, Jichi F, Ommen SR, Christiaans I, Arbustini E, Garcia-Pavia P, et al.. International external validation study of the 2014 European Society of Cardiology guidelines on sudden cardiac death prevention in hypertrophic cardiomyopathy (EVIDENCE-HCM). Circulation. 2018;137(10):1015-23.

28. Nakagawa S, Okada A, Nishimura K, Hamatani Y, Amano M, Takahama H, et al. Validation of the 2014 European Society of Cardiology sudden cardiac death risk prediction model among various phenotypes in Japanese patients with hypertrophic cardiomyopathy. Am J Cardiol. 2018;122(11):1939-46.

29. Maron BJ, Casey SA, Chan RH, Garberich RF, Rowin EJ, Maron MS. Independent assessment of the European Society of Cardiology sudden death risk model for hypertrophic cardiomyopathy. Am J Cardiol. 2015;116(5):757-64.

30. Leong KMW, Chow JJ, Ng FS, Falaschetti E, Qureshi N, Koa-Wing M, et al. Comparison of the prognostic usefulness of the European Society of Cardiology and American Heart Association/American College of Cardiology Foundation risk stratification systems for patients with hypertrophic cardiomyopathy. Am J Cardiol. 2018;121(3):349-55.

31. Desai MY, Smedira NG, Dhillon A, Masri A, Wazni O, Kanj M, et al. Prediction of sudden death risk in obstructive hypertrophic cardiomyopathy: potential for refinement of current criteria. J Thorac Cardiovasc Surg. 2018; 156(2):750-9.

32. Reis L, Teixeira R, Fernandes A, Almeida I, Madeira M, Silva J, et al. Prevention of sudden cardiac death in hypertrophic cardiomyopathy: what has changed in the guidelines? Arq Bras Cardiol. 2018;110(6):524-31.

33. O'Mahony C, Akhtar MM, Anastasiou Z, Guttmann OP, Vriesendorp PA, Michels M, et al. Effectiveness of the 2014 European Society of Cardiology guideline on sudden cardiac death in hypertrophic cardiomyopathy: a systematic review and meta-analysis. Heart. 2019;105(8):623-31. 